\title{
Trial results fail to support a role for B vitamins in the prevention of recurrent stroke
}

T he first randomized, placebocontrolled trial of B vitamin therapy for the prevention of major vascular events in patients with a history of stroke or transient ischemic attack (TIA) has been unable to show any benefit from such treatment. "The take-home message was that B vitamins were safe but they were not significantly more effective than placebo," says Graeme Hankey of Royal Perth Hospital, Australia, who codesigned and codirected the trial.

Elevated plasma levels of homocysteine are believed to confer an increased risk of stroke, myocardial infarction and vascular death. B vitamin therapy can lower levels of this amino acid in plasma, but trials in various patient groups have failed to show that such therapy has a significant preventative effect for major vascular events. Only one of these studies was conducted in patients with a history of stroke, however, and no placebo group was included in this trial. Thus, whether $B$ vitamins show a beneficial effect in such patients has remained unknown.

In the new study, named the VITATOPS trial, investigators randomly assigned 8,164 patients with recent stroke or TIA of the brain or eye-enrolled at one of 123 centers across 20 countries-to receive one tablet daily of B vitamins $(n=4,089)$ or placebo $(n=4,075)$. The $\mathrm{B}$ vitamin dose administered to the patients comprised $2 \mathrm{mg}$ folic acid, $25 \mathrm{mg}$ vitamin $\mathrm{B}_{6}$ and $0.5 \mathrm{mg}$ vitamin $\mathrm{B}_{12}$.

The trial participants were followed up every 6 months until the study's completion. The primary end point of the trial was the combined measure of nonfatal stroke, nonfatal myocardial infarction, or death from any vascular causes. All individuals originally assigned to receive either $B$ vitamin therapy or placebo were included in the primary outcome analysis.

At completion of the study, the trial participants had been followed up for a median period of 3.4 years. Overall, $616(15 \%)$ of the patients who received B vitamin therapy and 678 (17\%) of the patients who received placebo met the primary end point (relative risk [RR] 0.91, 95\% CI 0.82-1.00, $P=0.05$ ).

As with the primary outcome, few differences were observed in the secondary outcomes between the two treatment groups; that is, B vitamin therapy was not associated with significant reductions in RR of nonfatal or fatal stroke, nonfatal or fatal myocardial infarcation, or death from any cause. One exception to this pattern concerned death from vascular causes, the frequency of which was markedly lower with B vitamin treatment than with placebo.

In spite of the inability of B vitamins to reduce the incidence of the primary outcome and most of the secondary outcomes, assessment of a subset of patients, from whom blood samples were taken at baseline and at the final follow-up, confirmed that such treatment lowered homocysteine levels to the extent hypothesized by the investigators. This effect was shown to be consistent across various ethnic groups.

In terms of safety, B vitamin therapy was not associated with any unexpected adverse effects of a serious nature. Moreover, the frequencies of the most commonly reported adverse effects were similar in the B vitamin and placebo treatment groups.

According to the researchers, the results showed that $B$ vitamin therapy, while safe, was no more effective than placebo for preventing major vascular events in people with recent stroke or TIA. The outcome of a meta-analysis conducted by the investigators, which examined the new data alongside the results of previous randomized controlled trials of homocysteinelowering therapies, seemed to concur with this conclusion.

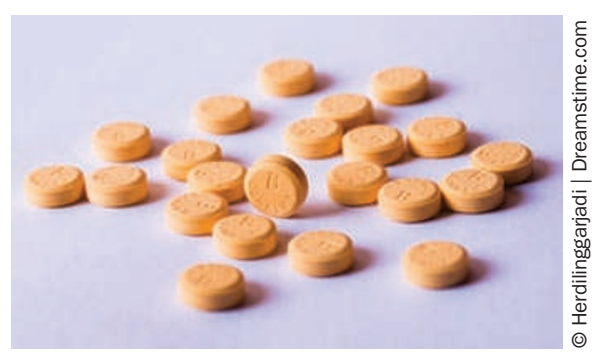

Despite the negative results of both the trial and the meta-analysis, a beneficial role for B vitamin therapy in the prevention of major vascular events in patients with a history of stroke could not be completely ruled out. Indeed, a subgroup analysis indicated that $B$ vitamins might reduce the risk of such events in patients with cerebral smallvessel disease that has caused lacunar infarction or intracerebral hemorrhage. Furthermore, as noted by the researchers, this study was only sufficiently powered to detect or reject a $15 \% \mathrm{RR}$ reduction in the primary outcome with B vitamin therapy, so any effect below this threshold would have been missed.

In their paper, the researchers state that the results of the VITATOPS trial do not support the use of B vitamins to prevent recurrent stroke. Hankey believes, however, that the end of the road has not yet been reached for B vitamin therapy. "We need to see the effect of $B$ vitamins in the three other ongoing trials, particularly in patients who have been treated for several years, and also in patients with symptomatic small-vessel disease of the brain."

Darran Yates

Original article The VITATOPS Trial Study Group. $B$ vitamins in patients with recent transient ischaemic attack or stroke in the VITAmins TO Prevent Stroke (VITATOPS) trial: a randomised, double-blind, parallel, placebo-controlled trial. Lancet Neurol. 9, 855-865 (2010) 